\title{
Variation of Molecular Mean Free Path in Confined Geometries
}

\author{
Jianfei Xie ${ }^{1, a)}$, Livio Gibelli ${ }^{2}$, Matthew K. Borg ${ }^{1}$, Oliver Henrich ${ }^{3}$, \\ Duncan A. Lockerby ${ }^{2}$ and Jason M. Reese ${ }^{1}$ \\ ${ }^{1}$ School of Engineering, University of Edinburgh, Edinburgh EH9 3WD, UK \\ ${ }^{2}$ School of Engineering, University of Warwick, Coventry CV4 7AL, UK \\ ${ }^{3}$ Department of Physics, SUPA, University of Strathclyde, Glasgow G4 ONG, UK \\ ${ }^{a}$ Corresponding author: jianfei.xie@ed.ac.uk
}

\begin{abstract}
This paper aims to settle disputes in the literature about the spatial variation of the molecular mean free path (MFP) in confined geometries. The MFP of a gas is determined by using both molecular dynamics (MD) and the direct simulation Monte Carlo (DSMC) technique. In spatially-homogeneous cases, the numerical results exactly recover the kinetic theory predictions of a constant MFP. However, in microchannels, the MFP is found to vary near to the bounding walls and reduce at the surfaces to half of its bulk value as long as collisions between gas molecules and wall atoms are taken into account in the calculation of the MFP.
\end{abstract}

\section{INTRODUCTION}

The molecular mean free path (MFP) is a key concept in transport phenomena in gases; it is defined as the average distance travelled by gas molecules between two successive collisions. The ratio of the MFP to the macroscopic characteristic length of the flow is referred to as the Knudsen number $(\mathrm{Kn})$, and the dynamics of a gas is known to vary significantly depending on its value. The MFP can be modelled from elementary kinetic theory arguments $[1,2,3]$; for a dilute gas composed of hard sphere molecules, the MFP is given by:

$$
\lambda_{h}=\frac{m}{\sqrt{2} \pi d^{2} \rho}
$$

where $m$ is the molecular mass, $d$ is the molecular diameter, and $\rho$ is the density of the gas. Eq. (1) indicates that the MFP decreases as the gas density increases, while it is independent of the gas temperature. A general expression for the MFP of gases in terms of viscosity is given by [2]:

$$
\lambda_{v}=\frac{\mu}{\rho} \sqrt{\frac{\pi m}{2 k_{B} T}},
$$

where $k_{B}$ is the Boltzmann constant, $\mu$ is the viscosity, and $T$ is the temperature of the gas. The above expressions for the MFP assume that the gas is dilute and spatially homogeneous, and that the molecules have a Maxwellian distribution function. However, for spatially-inhomogeneous cases, when a gas is bounded by a wall or surface, these expressions become questionable.

Stops [4] derived a space-dependent expression for the MFP of molecules leaving a wall, distributed according to the diffusive Lambert's cosine law of reflection. Several decades later, Guo et al. [5] extended the Stops model to propose the MFP of confined gases, $\lambda_{G}$, as

$$
\frac{\lambda_{G}}{\lambda_{0}}=1-\frac{1}{2}\left[y^{2} E_{1}(y)+(1-y) e^{-y}\right],
$$


where $y=z / \lambda_{0}, z$ is the distance from the wall, $\lambda_{0}$ is the nominal MFP in the bulk of the gas, and $E_{1}(y)$ is the exponential integral function, i.e. $E_{1}(y)=\int_{y}^{\infty} e^{-z} / z \mathrm{~d} z$. Recently, Abramov [6] introduced a new expression for the MFP in confined geometries by replacing the cosine law with the equilibrium Maxwellian to give:

$$
\frac{\lambda_{A}}{\lambda_{0}}=1-\frac{1}{2}\left[e^{-y}-y E_{1}(y)\right] .
$$

Beside its kinetic-theoretical interest, a space-dependent expression for the MFP, such as Eqs. (3) and (4), could be of practical relevance and has been used to propose scaling laws for the viscosity of a gas in the near-wall region $[5,6]$.

There have been a number of recent studies in which molecular dynamics (MD) simulations have been used to directly evaluate the MFP in a gas by averaging the recorded individual free paths $[7,8]$. However, there is still debate about the behaviour of the MFP in confined geometries. Some studies take into account the collisions between the freely moving gas molecules and wall atoms, as these are momentum-changing events, and show that the MFP varies near surfaces $[7,8]$. Others argue that the collisions between the freely moving gas molecules and wall atoms or adsorbed gas molecules should be disregarded when evaluating the individual free paths, and then show that the boundary-independent MFP given by Eq. (2) is recovered [9].

In the present paper, the MFP of gas molecules is numerically determined using both MD and the direct simulation Monte Carlo (DSMC) technique. Our aim is twofold. First, to resolve disputes in the literature about the spatial variation of the MFP for gases in confined geometries. Second, to assess the accuracy of the theoretical predictions proposed so far, i.e. Eqs. (3) and (4). Note that DSMC has not been used until now for this kind of investigation.

The rest of the paper is organised as follows. In Section 2, the MD and DSMC approaches are presented, and the procedures for the direct determination of the MFP are discussed. In Section 3, the MFP is numerically evaluated in the spatially-homogeneous case and in microchannels, and our simulation results are compared with the kinetic theory predictions. Conclusions are drawn in Section 4.

\section{METHODOLOGY}

\section{Molecular Dynamics Simulations}

MD can be used to measure directly the distances travelled by individual molecules in an ensemble between every collision. Monatomic gas molecules are modelled as interacting through a Lennard-Jones (LJ) 12-6 potential:

$$
U^{L J}\left(r_{i j}\right)= \begin{cases}4 \varepsilon\left[\left(\frac{\sigma}{r_{i j}}\right)^{12}-\left(\frac{\sigma}{r_{i j}}\right)^{6}\right], & r_{i j} \leq r_{c}, \\ 0, & r_{i j}>r_{c},\end{cases}
$$

where $U^{L J}$ is the intermolecular potential, $r_{i j}$ is the intermolecular distance, $\varepsilon$ is the energy parameter, $\sigma$ is the molecular diameter, and $r_{c}$ is the user-specified cutoff distance. Argon (Ar) molecules are used in our MD simulations, with $\sigma_{A r}=3.405 \times 10^{-10} \mathrm{~m}$ and $\varepsilon_{A r}=1.67 \times 10^{-21} \mathrm{~J}[10,11]$. In the case of a gas confined in a microchannel, we choose the wall atoms to be platinum (Pt), and the gas-wall (argon-platinum) interaction is also modelled with the Lennard-Jones potential, for which $\sigma_{A r-P t}=3.085 \times 10^{-10} \mathrm{~m}$ and $\varepsilon_{A r-P t}=0.894 \times 10^{-21} \mathrm{~J}$ are obtained from Lorentz-Berthelot mixing rules [12]. The molecular masses of argon and platinum are $m_{A r}=6.63 \times 10^{-26} \mathrm{~kg}$ and $m_{P t}=3.24 \times 10^{-25}$ $\mathrm{kg}$, respectively. The open source code LAMMPS (http://lammps.sandia.gov) [13] is used to perform the MD simulations in this paper, and a new class has been developed to calculate the individual free paths. The characteristic time unit in the MD simulations is chosen as $\tau=\sigma_{A r} \sqrt{m_{A r} / \varepsilon_{A r}}=2.15 \times 10^{-12} \mathrm{~s}$. The molecules move according to Newton's second law, and the equations of motion are integrated using a velocity Verlet algorithm with a time step of $\Delta t=0.002 \tau$. The potential is truncated at a cutoff of $r_{c}=2.5 \sigma_{A r}$, and the neighbour list method is adopted to reduce the time-consuming calculation of intermolecular interactions [12]. A Nosé-Hoover thermostat is applied to all gas molecules only in the initial part of the simulation until the system reaches an equilibrium state (i.e. typically the first 1,500,000 time steps in our MD simulations); the thermostat is then switched off while the individual free paths are recorded, so that the thermostat does not affect the dynamics. We spend about 2,000,000 additional time steps obtaining averaged macroscopic properties and the MFP.

In the case of bounding solid surfaces, to maintain a well-defined surface structure with a minimum number of solid atoms, each wall atom is tethered to a face-centred cubic (FCC) lattice site with a harmonic spring; it vibrates 




FIGURE 1. Schematic of the calculation of individual free paths in (a) molecular dynamics (MD) simulations, and (b) the direct simulation Monte Carlo (DSMC) technique. In (a), the parameters $l$ and $v$ are the individual free path and the velocity of a molecule, respectively, $r_{i j}$ is the distance between two molecules, $\sigma_{c o l}$ is the collision diameter, which is used to determine the collision event, $\Delta t$ is the time step in MD, and $n$ is the number of time steps between two successive collisions of the $i$ th molecule. In (b), for DSMC, $\Delta t, n, l$ and $v$ are similarly defined, except that near-neighbour collisions occur probabilistically between particles inside their current DSMC cell. Once a collision occurs, the free paths of the two particles are terminated and assigned to that same cell.

around its site at the Einstein frequency [14]. A velocity rescaling thermostat is applied to all wall molecules at the same temperature as the gas throughout the MD simulation. All MD simulations are run at STP. For example, there are 137,952 gas molecules in a $20 \lambda_{0}$ height microchannel $\left(\lambda_{0} \times \lambda_{0} \times 20 \lambda_{0}\right)$, which corresponds to $\mathrm{Kn}=0.05$. As $\mathrm{Kn}$ increases (or the channel height decreases), the number of gas molecules decreases accordingly. The number of wall atoms has been kept the same for all $\mathrm{Kn}$, and four layers of wall atoms consisting of 358,688 platinum atoms for each solid wall have been used in our MD simulations. This thickness of solid wall can guarantee that the influence of the fourth layer of wall atoms on our calculations of the free paths is negligible.

Our measurement of individual free paths in MD is illustrated in Fig. 1a). We set a condition to judge the occurrence of a collision event: if the distance between two gas molecules is equal to or less than the collision diameter, i.e. $r_{i j} \leq \sigma_{c o l}$, where $\sigma_{c o l}$ is the collision diameter, the two molecules have collided and we stop recording the free paths of the involved molecules. Because the continuous LJ potential is used, the two molecules may still move closer to each other and the distance between them can become smaller than the collision diameter, i.e. $r_{i j}<\sigma_{c o l}$. The finite time spent during collisions should be excluded when calculating the individual free paths, so we therefore restart recording the free paths when the distance between the molecules is again larger than the collision diameter, i.e. when $r_{i j}>\sigma_{c o l}$. In this procedure, the counter for recording the individual molecular free path is switched on after the last collision between two molecules and it is not switched off until the next collision.

\section{Direct Simulation Monte Carlo Approach}

The DSMC method was initially introduced for gas simulations based on physical arguments [15], but it has been proved to converge, in a suitable limit, to the solution of the Boltzmann equation [16]. The basic idea of DSMC consists in representing the velocity distribution function of the gas molecules by a number of computational particles. Particle motion and interactions are decoupled over a time step $\Delta t$ which is smaller than the local mean free time. The space domain to be simulated is covered by a mesh of cells whose size $\Delta x$ is less than the MFP. These cells are used to collect together particles that may collide according to stochastic rules derived from the Boltzmann equation. The cells employed for simulating interactions are also used for the sampling of macroscopic properties, which are obtained through weighted averages of the particle properties.

In the present work, particles' free paths are evaluated in a similar way to MD, by tagging particles and keeping track of their free flight distance by an appropriate counter that measures the distance incremental by a particle in that time step. See Fig. 1b); when particles collide, the counter is set to zero no matter whether the collision occurred with a wall or with another particle. As particle-particle collisions occur in a DSMC cell, so the free path is collected and assigned to the same cell for further post-processing. Our numerical experiments have shown that the MFP turns out to be discontinuous if the free flights of the molecules hitting the walls are attributed to the cells closest to them. The 

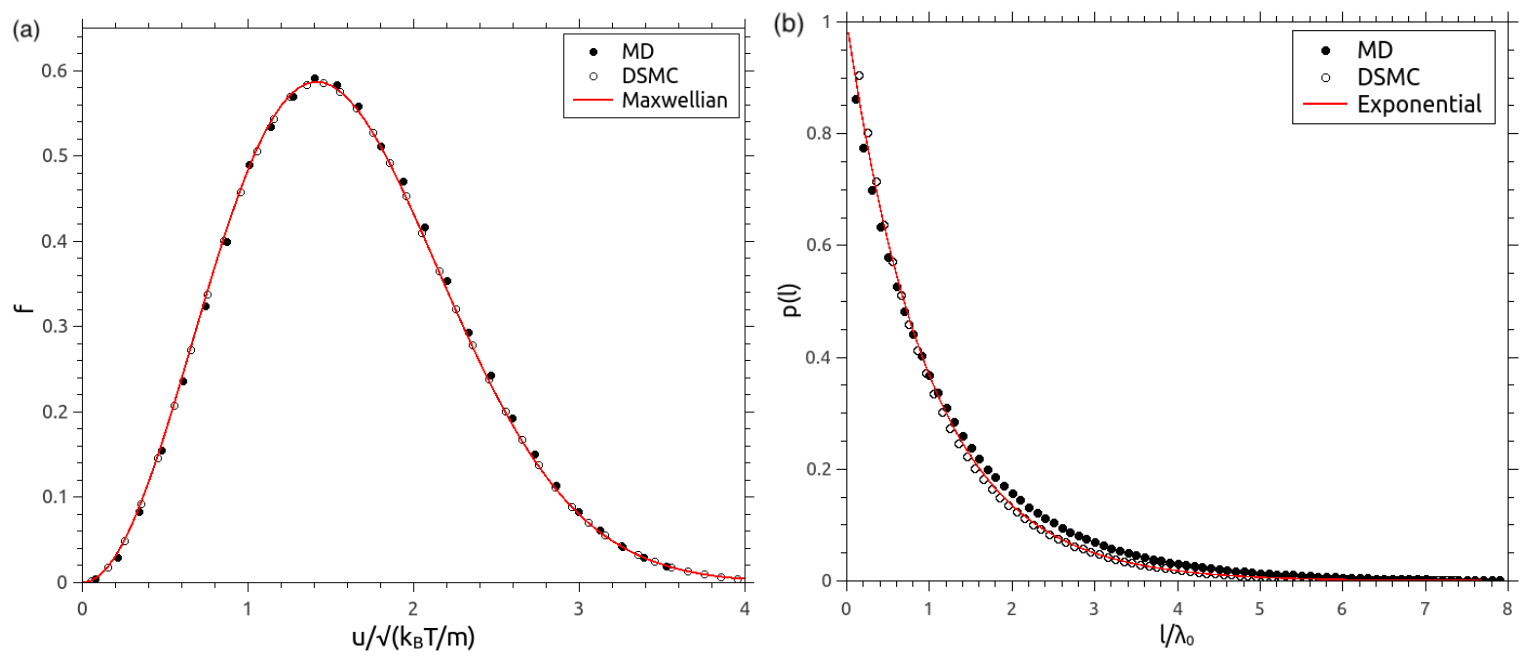

FIGURE 2. (a) Normalised velocity distribution function of the simulated gas molecules. The molecular velocity $u$ is normalised by $\sqrt{k_{B} T / m}$, where $k_{B}$ is the Boltzmann constant, $T$ is the gas temperature, and $m$ is the molecular mass. (b) Probability density distribution of the individual free paths of gas molecules; $p(l)$ is the probability density of free paths, and $\lambda_{0}$ is the nominal MFP.

continuity of the MFP is recovered by setting to zero the counter of molecules which suffer a collision with walls but disregarding their free flight contribution.

The simulations we report below have been obtained by setting $\Delta x=10^{-2} \lambda_{h}$ and $\Delta t=2 \times 10^{-3} t_{0}$, where $t_{0}=\lambda_{h} /\left(k_{B} T_{0} / m\right)$, and $\lambda_{h}$ and $T_{0}$ are the nominal mean free path and a reference temperature, respectively. Computational particles are initially distributed according to a Maxwellian at the temperature $T_{0}$ and their average number per cell during simulations is about $10^{2}$. The evolution of the system is simulated for $10^{3} t_{0}$. As an input parameter for the boundary condition, both fully diffusive wall (i.e. the tangential momentum accommodation coefficient is equal to one) and a mixture of partially diffusive and partially specular walls (i.e. the tangential momentum accommodation coefficient is less than one) have been used in our DSMC simulations: four values of the tangential momentum accommodation coefficient (i.e. 0.2, 0.4, 0.6, 0.8 and 1.0) are tested. Our results show that the influence of the tangential momentum accommodation coefficient on the calculations of MFP is negligible. For the sake of simplicity, the fully diffusive wall is adopted in all our DSMC simulations.

\section{RESULTS AND DISCUSSION}

\section{Spatially Homogeneous Case}

We first consider the MFP of argon molecules contained in a cubic spatial domain with periodic boundary conditions in all directions, with the length of each side of the cube set to about one MFP given by Eq. (2). After equilibration of the system, the calculated velocity distribution function and free path distribution of the gas molecules are shown in Figs. 2 (a) and (b), respectively. As expected, in both the MD and the DSMC simulations, the gas molecules are distributed according to a Maxwellian, and the probability density of the free path distribution is exponential, i.e. of the form $p(l)=\lambda_{0}{ }^{-1} \exp \left(-l / \lambda_{0}\right)$, where $l$ is the individual free path. It should be noted that the domain of the system should be at least one mean free path in extent, to keep the gas molecules in local thermodynamic equilibrium.

As detailed in the previous section, in order to directly evaluate the MFP in MD simulations, a procedure for judging the collision event between two molecules is required, including the choice of a collision diameter. For argon molecules, Dongari et al. [7] suggested $\sigma_{c o l}=1.0 \sigma_{A r}$, while Barisik and Beskok [9] used $\sigma_{c o l}=1.06 \sigma_{A r}$ by combining the basic concept of MFP for hard spheres in Eq. (1) and the viscosity-based MFP in Eq. (2). To et al. [8] adopted $\sigma_{c o l}=1.014 \sigma_{A r}$ as a best fit of the predictions of kinetic theory to their calculations of MFP using MD. In order to recover the kinetic theory prediction of the MFP, i.e. Eq. (2), a temperature-dependent collision diameter is proposed to determine the collision events between the gas molecules. Fig. 3 (a) shows the variation of MFP as a function of the collision diameter at a low gas temperature (i.e. $273 \mathrm{~K}$ ). The MD-measured MFP decreases with the increase of the 

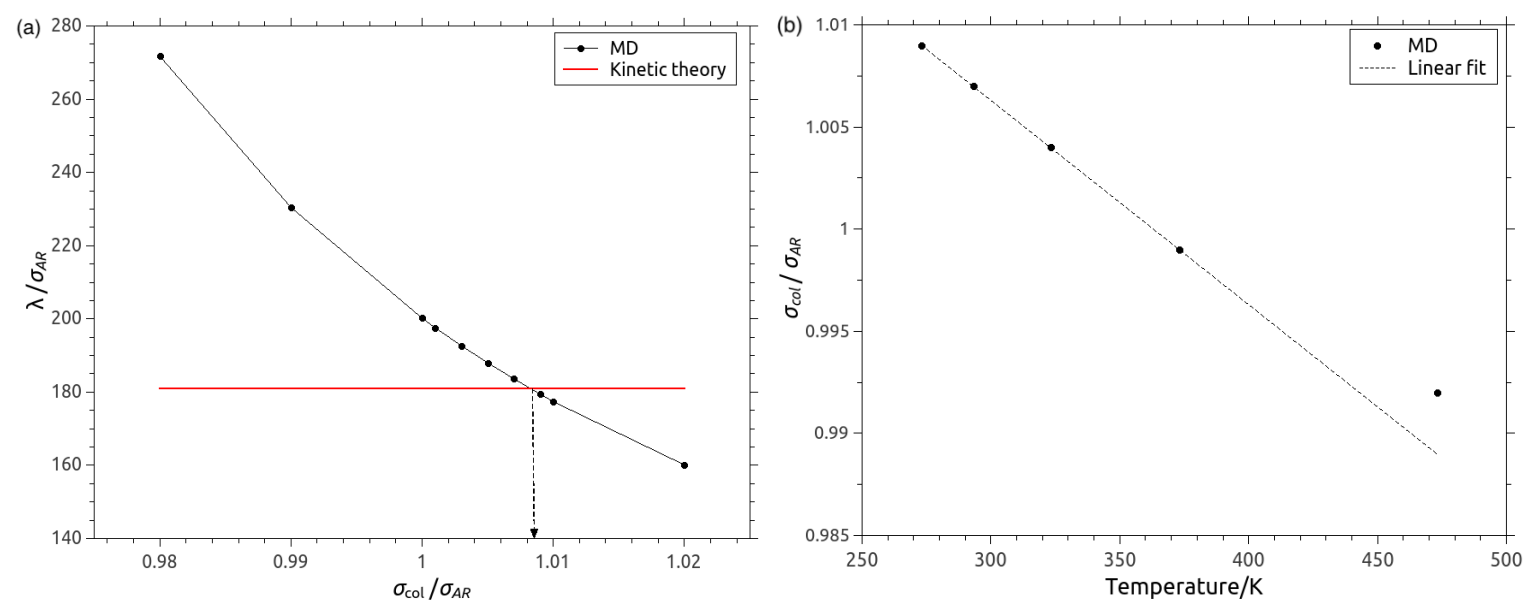

FIGURE 3. (a) A calibration study of the collision diameter $\sigma_{c o l}$ for argon in a spatially-homogeneous system at $T=273 \mathrm{~K}$. We measure the MFP in MD simulations for varying prescribed $\sigma_{c o l}$ (filled circles). The black solid line is the MD results, and the red solid line is the prediction of kinetic theory, Eq. (3). In this case, the calibrated collision diameter is $\sigma_{c o l}=1.0089 \sigma_{A r}$. (b) Temperature-dependent collision diameter from the calibration procedure. The black dashed line is a linear fit to the calibrated collision diameters at low gas temperatures (up to $373 \mathrm{~K}$ ), with $\sigma_{c o l}(T) / \sigma_{A r}=1.0363-0.0001 T$.

collision diameter, and the intersection with the prediction of kinetic theory is when $\sigma_{c o l}=1.008 \sigma_{A r}$. Likewise, the approximate collision diameter can be determined over a range of temperatures, and the results are shown in Fig. 3 (b). The collision diameter decreases as the gas temperature increases, and in the range of explored temperatures a linear relationship can be estimated, i.e. $\sigma_{c o l}(T) / \sigma_{A r}=1.0363-0.0001 T$ with $T$ up to $373 \mathrm{~K}$. As the gas temperature increases $(\sim 473 \mathrm{~K})$, the decrease of collision diameter becomes less pronounced (i.e. a slight deviation from the linear fit). This temperature-dependent collision diameter can guarantee good agreement of the MFPs determined by MD simulations with the predictions of kinetic theory for gas temperatures in the range of $273 \mathrm{~K}$ to approximately $473 \mathrm{~K}$.

\section{Spatially Inhomogeneous Case}

We now consider a gas confined in a microchannel, in equilibrium at STP, as shown in Fig. 4 (a). The two solid walls are parallel to the $x y$ plane, with periodic boundary conditions imposed along the $x$ and $y$ directions. The distance between the two parallel walls in the $z$ direction is $H$. Note that in our MD simulations the solid walls consist of platinum atoms forming two planes of a face-centred cubic (FCC) lattice, and the interactions between gas and wall are modelled by Lennard-Jones potentials [17] (and there is no gas adsorption). By contrast, in DSMC the gas-wall interactions are dealt with by the Maxwellian scattering kernel with complete accommodation [2]. In both approaches, the spatial variation of MFP is obtained by assigning the individual free paths to small bins that divide the space in the $z$ direction [7, 8]. As the collisions between gas molecules and wall atoms are momentum-changing events, we have taken these into account when recording the free paths; however, we note that a different free-path definition that does not include them is also potentially valid. We investigate the impact of such a choice below.

As can be seen in Fig. 4 (b), the measured MFP varies near the wall, and at the wall is half its nominal bulk value. The decrease of the MFP in this near-wall zone becomes greater with increasing Kn (where Kn can be changed by changing the channel height $H$ ). At small $\mathrm{Kn}$, the MD determination of the MFP variation agrees well with the DSMC results and Abramov's prediction [6], i.e. Eq. (4). The expression of Guo et al. [5], i.e. Eq. (3), somewhat underpredicts the MFP near the walls. To et al. [8] obtained a MFP of zero near the wall in their MD simulations for $\mathrm{Kn}=0.18$ and 0.37 , but they only considered molecules outgoing from the walls.

As mentioned, some previous studies have argued that the MFP should be defined as the averaged distance travelled by gas molecules between two successive collisions with other gas molecules only, and that collisions between gas molecules and wall atoms or gas molecules adsorbed on the surface should not be taken into account when evaluating individual free paths [9]. Using such a definition, a constant and isotropic MFP would be expected. In order to assess this, we have run MD and DSMC simulations in a microchannel $\left(H=20 \lambda_{0}\right)$ and disregarded gas-wall collisions in the evaluation of the MFP. While interactions between gas molecules and wall atoms are still part of the dynamics, 

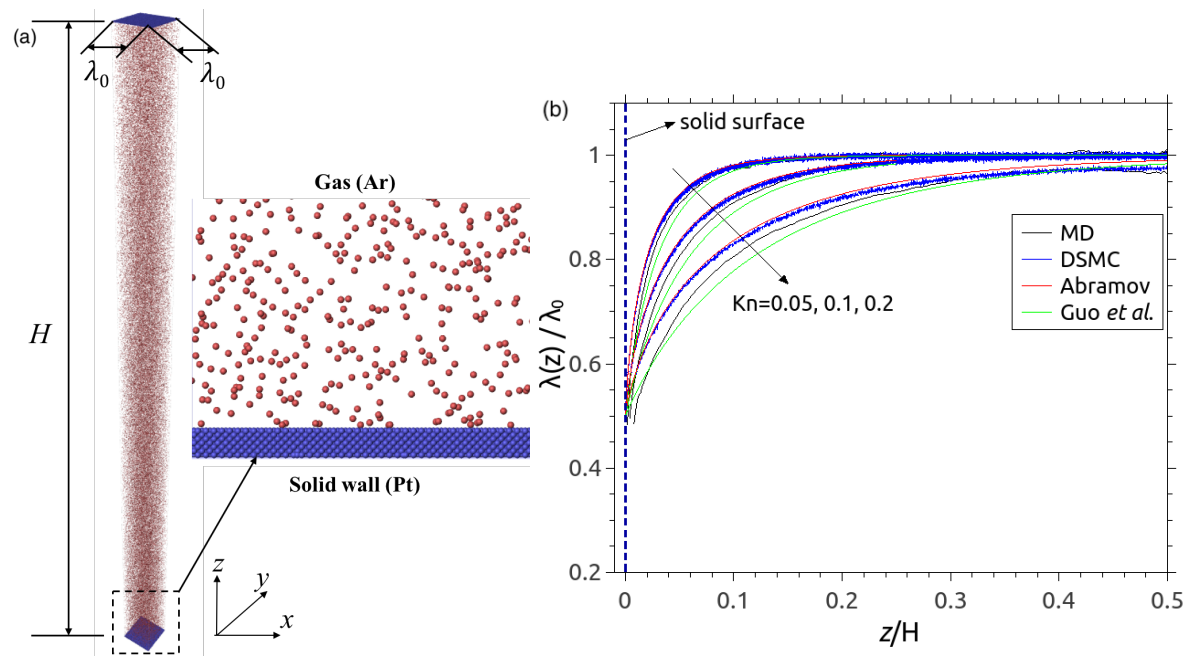

FIGURE 4. (a) A gas in equilibrium confined in a microchannel at standard temperature and pressure (STP). The two solid atomic walls are parallel to the $x y$ plane, and periodic boundary conditions are imposed along the $x$ and $y$ axis. The distance between the two parallel walls in the $z$ direction is $H$. (b) Spatial variation of the normalised MFP in microchannels; channel heights of $H=20 \lambda_{0}, 10 \lambda_{0}$ and $5 \lambda_{0}$ are simulated in order to produce $\mathrm{Kn}=0.05,0.1$ and 0.2 , respectively. The data is plotted for only half the channel (i.e. above one of the surfaces).

the individual free paths are not terminated at the walls. Our simulations show that a constant MFP is predicted by both MD and DSMC simulations when the walls are perfectly specular, and Fig. 5 shows that a constant MFP is also obtained when real molecular (MD) and fully diffusive (DSMC) walls are used. Compared to the decrease of MFP at the walls when gas-wall collisions are counted, both MD and DSMC predict a constant MFP when gas-wall collisions are disregarded, whatever kind of walls (i.e. specular or diffusive) we utilise.

The results clearly show that the variation of the MFP near to walls is not inconsistent with kinetic theory. As already mentioned, classical expressions of the MFP, i.e. Eqs. (1) and (2), only refer to a spatially homogeneous gas. Collisions between the gas and bounding surfaces can be encompassed in the concept of the MFP, and the resulting spatial variation of the MFP may help in understanding transport in Knudsen layers near surfaces.

\section{CONCLUSIONS}

The molecular mean free path (MFP) in a gas has been evaluated using both molecular dynamics (MD) and the direct simulation Monte Carlo (DSMC) technique. The novelty of this paper is threefold:

i) we have shown that in microchannels (spatially-inhomogeneous cases) the MFP at the walls reduces smoothly to half of its bulk value as long as the collisions between the gas and the wall are accounted for. A wall-distance dependent MFP is therefore not a numerical artefact but rather a consequence of taking into account the collisions between gas and wall molecules in the calculation of the MFP. Our results are not in disagreement with elementary kinetic theory expressions for the MFP, as these expressions refer to a spatially homogeneous gas;

ii) we have assessed some of the theoretical scaling laws proposed in the literature $[4,5,6]$ so far; and

iii) we have found that DSMC is a suitable tool for direct evaluation of the MFP (quite surprisingly DSMC has not been used before for this kind of study).

We conclude by stressing that the answer to the question of which is the more appropriate definition of the MFP depends on the purpose for which the MFP is intended. If it is used to scale transport properties near walls $[5,6,7,8]$, then a definition that accounts for gas-wall collisions is needed.

\section{ACKNOWLEDGMENTS}

The authors acknowledge the support of the UK's Engineering and Physical Sciences Research Council (EPSRC) via grant nos. EP/N016602/1 and EP/R007438/1. JMR acknowledges the support of the Royal Academy of Engineering 


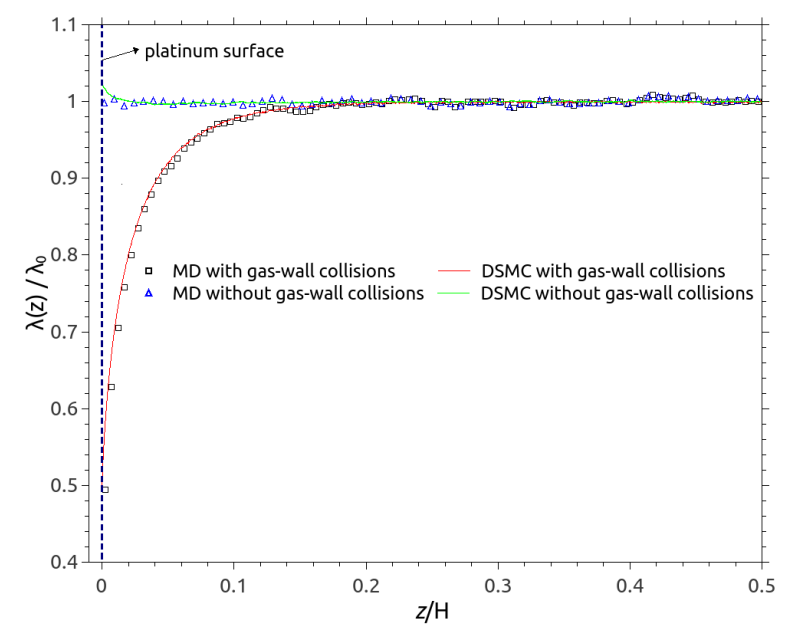

FIGURE 5. Spatial variation of the normalised MFP of the gas in a microchannel $\left(H=20 \lambda_{0}\right)$. Open squares (black) and triangles (blue) refer to the MD simulation results that count and do not count the gas-wall collisions, respectively; red and green solid lines refer to the DSMC simulation results that count and do not count the gas-wall collisions, respectively. The data is plotted for only half the channel (i.e. above one of the surfaces).

under the Chair in Emerging Technologies scheme. JFX thanks the National Natural Science Foundation of China (grant no. 51506110) for their support. OH acknowledges support from the EPSRC Early Career Research Software Engineer Fellowship Scheme (EP/N019180/2). The authors thank Dr Srinivasa Ramisetti for useful discussions.

\section{REFERENCES}

[1] E. H. Kennard, Kinetic Theory of Gases (McGraw-Hill Book Company, Inc., New York and London, 1938).

[2] C. Cercignani, Mathematical Methods in Kinetic Theory (Plenum Press, New York and London, 1990).

[3] G. M. Kremer, An Introduction to the Boltzmann Equation and Transport Processes in Gases (Springer, Berlin, Heidelberg, 2010).

[4] D. W. Stops, Journal of Physics D: Applied Physics 3, 685-696 (1970).

[5] Z. L. Guo, B. C. Shi, and C. G. Zheng, Europhys. Lett. 80, p. 24001 (2007).

[6] R. V. Abramov, Journal of Nonlinear Science 28, 833-845 (2018).

[7] N. Dongari, Y. Zhang, and J. M. Reese, Journal of Physics D: Applied Physics 44, p. 125502 (2011).

[8] Q. D. To, C. Léonard, and G. Lauriat, Phys. Rev. E 91, p. 023015 (2015).

[9] M. Barisik and A. Beskok, Microfluidics and Nanofluidics 18, 1365-1371 (2015).

[10] J. O. Hirschfelder, C. F. Curtiss, and R. B. Bird, Molecular Theory of Gases and Liquids (John Wiley \& Sons, Inc., New York, 1964).

[11] J.-F. Xie and B.-Y. Cao, Molecular Simulation 43, 65-75 (2017).

[12] M. P. Allen and D. J. Tildesley, Computer Simulation of Liquids (Clarendon, Oxford, 1987).

[13] S. Plimpton, J. Compt. Phys. 117, 1-19 (1995).

[14] B. Y. Cao, M. Chen, and Z. Y. Guo, Applied Physics Letters 86, p. 091905 (2005).

[15] G. A. Bird, Molecular Gas Dynamics and the Direct Simulation of Gas Flows (Oxford University Press, Oxford, 1994).

[16] W. Wagner, Journal of Statistical Physics 66, 1011-1044 (1992).

[17] J.-F. Xie and B.-Y. Cao, Computers \& Fluids 160, 42-50 (2018). 